\title{
Metastatic Cervical Adenosquamous Carcinoma
}

National Cancer Institute

\section{Source}

National Cancer Institute. Metastatic Cervical Adenosquamous Carcinoma. NCI

Thesaurus. Code C153390.

A cervical adenosquamous carcinoma that has spread to another anatomic site. 